\title{
Analyzation and Understanding of Final Rule on Post-marketing Safety Reporting of Combination Products
}

\section{Gill A* and Talla VK}

Dr. Reddy's Laboratories Ltd., Princeton, NJ, USA

\section{Introduction}

As per (21 CFR part 3) combination product is composed of two or more different types of medical products; a combination of a drug, device, and/or biological product with one another. "Constituent parts" of the combination product are the drugs, devices, and biological products included in combination products

Physically, chemically or otherwise combined products as per (21 CFR 3.2(e) (1)) are:

- Monoclonal antibody and a therapeutic drug combined.

- Device coated or impregnated with a drug or biologic. Such as: catheter with antimicrobial coating, condom with spermicide or biologic coatings in orthopedic implants.

- Prefilled syringes, transdermal patches, insulin injector pens and metered dose inhalers.

Combination products with components packaged together as per (21 CFR 3.2(e) (2)) are:

- Drug or biological product packaged with a delivery device.

- Surgical tray with surgical instruments, drapes, and lidocaine or alcohol swabs.

Combination products with components provided separately but are labeled for use together (21 CFR 3.2(e) (3) or (e) (4)):

- Photosensitizing drug and activating laser/light source.

- Iontophoretic drug delivery patch and controller.

Effective January 19, 2017, FDA issued a final rule on reporting of combination products that all applicants subject to this rule be in compliance with existing provisions based on their filing status. The rule implementation is within the 18 months from the rule effective date for the new requirements of $\$ 4.102(\mathrm{c})$ and (d) for combination product, of $\$ \$ 4.103$ and 4.105 (a) (2) for constituent part, and of $\$ \$ 4.104(\mathrm{~b})$ and 4.105 (b) for combination product applicants.

\section{Objective}

To understand and analyze the newly issued FDA final rule on postmarketing reporting of Combination Products and how the unique reporting requirements of constituent parts of combination products may result in under reporting or confusion for combination products.

\section{Overview}

In the past FDA had not issued specific regulations on safety reporting for combination products in post-marketing setting. In the final rule, the agency employed specifications to combination products, applicable to constituent's parts, from the post-marketing safety reporting regulations. The regulations for drug, devices and biological properties have similarities but also has unique reporting requirements such as reporting timeframes. Lack of clarity on how to employ different requirements and regulations on combination products may result in under reporting and inconsistency for reporting of combination products and its constituent parts in post-marketing setting.

The agency's aim to propose the new rule was to bring consistency in post-marketing reporting and to avoid the duplicative reporting. As per the agency, due to the parallels in the post-marketing reporting of drugs, devices and biological products safety, relying on and complying with existing rules seemed to be the best approach when considering the rule. Entities subject to the proposed rule included those subject to PMSR duties under 21CFR parts 314 (drugs), 600 and 606 (biological products) and 803 (devices), except for user facilities and distributors as defined under part 803 [1,2].

Previously, the similarities between device, drug and biological products include reporting of serious adverse events, death and life threatening reports, provision for expedited and non-expedited reports and follow-ups. The differences in reporting, however, are more focused on different timelines when adverse events are reported for drugs, devices and biological products. For drugs, expedited reports are submitted by day 15 whereas for devices, manufactures have 30 days to report death, serious injuries and malfunctions.

The final rule presented the same approach as was in proposed rule but with simplifications, clarifications, addition, and other changes. The final rule applies necessary reporting requirements to combination products in post-marketing setting to ensure the safety and effectiveness of the product. It clarifies how to implement the reporting requirements applicable to combination products and enables efficiencies in cases where multiple reporting requirements may apply for an event and to submit a single report instead of multiple reports [3].

The final rule eradicates the requirement for combination products that got approved under an application other than Biologics License

*Corresponding author: Amrit Gill, Dr Reddy's Laboratories Ltd., Princeton, NJ, 08540, USA, Tel: 888-375-3784; E-mail: dr.amrit.gill@gmail.com

Received: June 05, 2017; Accepted June 20, 2017; Published June 27, 2017

Citation: Gill A, Talla VK (2017) Analyzation and Understanding of Final Rule on Post-marketing Safety Reporting of Combination Products. J Pharmacovigil 5: 230. doi:10.4172/2329-6887.1000230

Copyright: (c) 2016 Gill A, et al. This is an open-access article distributed under the terms of the Creative Commons Attribution License, which permits unrestricted use, distribution, and reproduction in any medium, provided the original author and source are credited. 
Application (BLA) to comply with blood fatality reporting requirements as described in $\$ 606.170$. With the final rule, for combination products that got approval under a device application will now be allowed to submit 15 -day reports as described in $\$ \$ 314.80$ and 600.80 within 30 days instead of 15 days.

Few questions arise from the final rule on Post-marketing Safety Reporting of Combination Products:

- Is this really beneficial for manufacturers?

- Is it going to create confusion to manufacturers that develop drugs, devices and combination products?

- Is it cost effective, long term and short term?

- Will the changes impact signal detection process in terms of comparing to legacy data?

- Harmonization with other regional requirements. EU, Japan Vs Emerging markets.

- Dealing with follow-up reports?

The answer to all of these questions is similar. Though the new process just like any other will create some confusion in the beginning, the agency has tried to provide a very clear and transparent roadmap for manufacturers' understanding of the new rule. The agency has provided ample enough time (18 months) to implement the final rule from its effective date. The agency clarified few comments from the industry in response to the proposed rule.

Having said that, there still are confusions about many aspects of this final rule. Industry is still trying to understand how will signal detection change? The legacy data on some of these products may have been under drug regulations only or device regulation. With the final rule, there seems to be no guidance on how is that legacy data going to be used for comparison to the new data.

Another point that the agency continues to state is that this rule is not intended to create new CGMP requirements, however, it intends to clarify how to apply them to combination products [4]. The confusion will also lie around manufacturers where they have different arrangements such as out-licensing/in-licensing or where (A) NDA holder is using a contract manufacturing organization. The agency clarified that both specification developers and contract manufacturers "manufacture" are considered manufacturers for purposes of these underlying CGMP regulations and are subject to the rule if they manufacture combination products or constituent parts of combination products. However, an entity that is not considered a manufacturer for purposes of these regulation, which manufactures a device component, is not subject to this rule even if that component will be incorporated into a combination product or constituent part of a combination product at some other facility [4].

Though the agency has tried to clarify few definitions such as "constituents part", the manufacturers feel that more clarification is needed. Confusion around what is subject to QS regulations and what is not still exists. The volume and complexity of the new requirements in the final rule may compromise rather than facilitate the agency's objectives for both the market authorization holders as well as agency. There is no clarity on harmonization between FDA and other regions when it comes to the final rule for those manufacturers who have market presence in different regions.
Another concern surrounding the final rule is processing of followup cases that may have been received and processed initially under the current regulations. How will the initial and follow-ups on the same case will be harmonized when those were processed with two different regulations.

As far as financial burden is concerned, in the short term, this rule will burden the manufacturers with one-time administrative costs from understanding the rule, evaluation of current compliance and procedures, adjustment of existing standards of practice, applying changes to the storage and reporting software, and training appropriate personnel (which may include in house staff or offsite vendor teams) on the requirements under this rule. Firms that do not currently observe the reporting requirements specified by the final rule will also acquire annual reporting costs from the submission of field alert reports, 5-day reports, malfunction reports, correction or removal reports, and biological product deviation reports, as applicable [5].

In the long term however, the organizations may be benefited in terms of decreased potentially duplicative reporting and more consistency. However, for some small pharmas, the short term administrative cost to implement these changes could be a big burden.

\section{Conclusion}

Industry understands and values FDA's efforts to provide clarity on these requirements, streamlining and reducing unnecessary reporting and improving the quality of reports, however, the confusion around some of the processes associated with the final rule on Post-marketing Safety Reporting of Combination products is concerning. High chances of additional workload for companies that have worldwide presence due to some of the changed not aligned with ICH guidelines, and industry practice. It is also believed that the cost to implement the new requirements may outweigh any savings realized through efficiency gains and redundant reporting for specifically many small organizations.

\section{References}

1. https://www.fda.gov/combinationproducts/aboutcombinationproducts/ ucm101496

2. https://www.federalregister.gov/d/2016-30485/p-49

3. https://www.federalregister.gov/d/2016-30485/p-55

4. https://www.fda.gov/downloads/combinationproducts/ucm336194.pdf

5. https://www.fda.gov/downloads/AboutFDA/ReportsManualsForms/Reports/ EconomicAnalyses/UCM533689.pdf 